\title{
Proinov type contractions on dislocated $b$-metric spaces
}

\author{
Badr Alqahtani ${ }^{1}$, Sara S. Alzaid ${ }^{1}$, Andreea Fulga² and Antonio Francisco Roldán López de Hierro ${ }^{3 *}$
}

"Correspondence: aroldan@ugr.es
${ }^{3}$ Department of Statistics and
Operations Research, University of
Granada, Facultad de Farmacia,
Campus de Cartuja, s/n, 18071,
Granada, Spain
Full list of author information is
available at the end of the article

available at the end of the article

\begin{abstract}
In this paper, we improve the Proinov theorem by adding certain rational expressions to the definition of the corresponding contractions. After that, we prove fixed point theorems for these modified Proinov contractions in the framework of dislocated $b$-metric spaces. We show some illustrative examples to indicate the validity of the main results.
\end{abstract}

Keywords: Fixed point theory; Simulation function; Contraction mapping

\section{Introduction and preliminaries}

In the nature of mathematics, there is the purpose of generalizing, expanding, and obtaining the most general forms of existing concepts and results. The concept of metric, which is the most fundamental and solid basis of the analysis study, has been constantly expanded and generalized with this motivation. Examples of the new metrics that have been put forward for this purpose can be counted as quasi-metric, $b$-metric, partial-metric, symmetric, D-metric, modular metric, fuzzy metric, soft-metric, G-metric, and so on. On the other hand, it was understood that not all of these newly defined metrics provide a new and original structure. For instance, G-metric can be reduced to semi-metric or cone metric to a standard metric. More examples can be given, but here we stop to focus on the main motivation. Two of the new and original generalizations of metric notions are $b$-metrics [1-16] and dislocated metrics [17-21]. Very recently, these two notions have emerged under the name of dislocated $b$-metric $[22,23]$.

Metric fixed point theory is a field of study that needs an abstract metric framework (see, for instance, [24-27]). Very recently Proinov [28] proved a fixed point theorem that not only unifies but also generalizes a number of well-known results in the framework of a standard metric space. In particular, he proved that Wardowski [29] and Jleli and Samet [30] results are not only equivalent to each other, but also they are a special case of one of the main results of [28].

In this paper, we improve the Proinov type contractions by involving certain rational expression to the corresponding contraction thought by Proinov [28]. After then, we prove fixed point theorems for these modified Proinov contractions in the framework of dislocated $b$-metrics. We bring forward illustrative examples to show the validity of the main results.

(c) The Author(s) 2021. This article is licensed under a Creative Commons Attribution 4.0 International License, which permits use sharing, adaptation, distribution and reproduction in any medium or format, as long as you give appropriate credit to the original author(s) and the source, provide a link to the Creative Commons licence, and indicate if changes were made. The images or other third party material in this article are included in the article's Creative Commons licence, unless indicated otherwise in a credit line to the material. If material is not included in the article's Creative Commons licence and your intended use is not permitted by statutory regulation or exceeds the permitted use, you will need to obtain permission directly from the copyright holder. To view a copy of this licence, visit http://creativecommons.org/licenses/by/4.0/. 
Let $\mathrm{S}$ be a nonempty set and $\mathbb{N}=\{1,2,3, \ldots\}$. Some examples of rational contractivity conditions are shown in the following results (see also [31]).

Theorem $1([32])$ Let $(\mathrm{S}, d)$ be a complete metric space and $z: \mathrm{S} \rightarrow \mathrm{S}$ be a mapping such that there exist $k_{1}, k_{2} \in[0,1)$ with $k_{1}+k_{2}<1$ such that

$$
d(Z \mathrm{v}, Z \mathrm{w}) \leq k_{1} \cdot d(\mathrm{w}, Z \mathrm{w}) \frac{1+d(\mathrm{v}, Z \mathrm{v})}{1+d(\mathrm{v}, \mathrm{w})}+k_{2} \cdot d(\mathrm{v}, \mathrm{w})
$$

for all $\mathrm{v}, \mathrm{w} \in \mathrm{S}$. Then $z$ has a unique fixed point $\mathrm{x} \in \mathrm{S}$, and the sequence $\left\{Z^{n} \mathrm{v}\right\}$ converges to the fixed point $\mathrm{x}$ for all $\mathrm{v} \in \mathrm{S}$.

Theorem 2 ([33]) Let $(\mathrm{S}, d)$ be a complete metric space and $\mathrm{Z}: \mathrm{S} \rightarrow \mathrm{S}$ be a continuous mapping. If there exist $k_{1}, k_{2} \in[0,1)$ with $k_{1}+k_{2}<1$ such that

$$
d(z \mathrm{v}, z \mathrm{w}) \leq k_{1} \cdot \frac{d(\mathrm{v}, Z \mathrm{v}) d(\mathrm{w}, Z \mathrm{w})}{d(\mathrm{v}, \mathrm{w})}+k_{2} \cdot d(\mathrm{v}, \mathrm{w})
$$

for all distinct $\mathrm{v}, \mathrm{w} \in \mathrm{S}$, then $\mathrm{Z}$ possesses a unique fixed point in $\mathrm{S}$.

Theorem 3 ([28]) Let $(\mathrm{S}, d)$ be a metric space and $z: \mathrm{S} \rightarrow \mathrm{S}$ be a mapping such that

$$
\Psi(d(z \mathrm{v}, z \mathrm{w})) \leq \Phi(d(\mathrm{v}, \mathrm{w}))
$$

for all $\mathrm{v}, \mathrm{w} \in \mathrm{S}$ with $d(Z \mathrm{v}, Z \mathrm{w})>0$, where the functions $\Psi, \Phi:(0, \infty) \rightarrow \mathbb{R}$ are such that the following conditions are satisfied:

1. $\Psi$ is nondecreasing;

2. $\Phi(\theta)<\Psi(\theta)$ for any $\theta>0$;

3. $\lim \sup _{\theta \rightarrow \theta_{0^{+}}} \Phi(\theta)<\Psi\left(\theta_{0}+\right)$ for any $\theta_{0}>0$.

Then $z$ admits a unique fixed point.

Definition 4 ([34]) A function $\mathrm{d}_{l}: \mathrm{S} \times \mathrm{S} \rightarrow[0, \infty)$ is a dislocated-metric on $\mathrm{S}$ if it satisfies the conditions:

$d_{l 1} . \mathrm{d}_{l}(\mathrm{v}, \mathrm{w})=0 \Rightarrow \mathrm{v}=\mathrm{w}$;

$d_{l 2}$. symmetry: $\mathrm{d}_{l}(\mathrm{w}, \mathrm{v})=\mathrm{d}_{l}(\mathrm{v}, \mathrm{w})$;

$d_{l 3}$. the triangle inequality

$$
\mathrm{d}_{l}(\mathrm{u}, \mathrm{w}) \leq \mathrm{d}_{l}(\mathrm{u}, \mathrm{v})+\mathrm{d}_{l}(\mathrm{v}, \mathrm{w})
$$

for all $\mathrm{u}, \mathrm{v}, \mathrm{w} \in \mathrm{S}$. In this case, the pair $\left(\mathrm{S}, d_{l}\right)$ is a dislocated-metric space (shortly $d_{l}$-MS).

Definition 5 ([35]) Let $s \in[1, \infty)$ be a real number. A function $b: S \times S \rightarrow[0, \infty)$ is a b-metric on $S$ if it satisfies the conditions:

$b_{1} . b(v, w)=0 \Leftrightarrow v=w$,

$b_{2}$. symmetry: $b(w, v)=b(v, w)$

$\mathrm{b}_{3}$. the generalized version of the triangle inequality involving the number $\mathrm{s}$

$$
b(u, w) \leq s[b(u, v)+b(v, w)] \text { for all } u, v, w \in S .
$$

In this case, the tripled $(\mathrm{S}, \mathrm{b}, \mathrm{s})$ forms a b-metric space (shortly b-MS). 
Obviously, for $\mathrm{s}=1$, we find the notion of metric space.

Definition 6 ([36]) Let $s \in[1, \infty)$ be a real number(given). A function $d_{b}: S \times S \rightarrow[0, \infty)$ is a dislocated $\mathrm{b}$-metric on $\mathrm{S}$ if it satisfies the conditions:

$$
\begin{array}{ll}
d_{b_{1}} . & d_{b}(v, w)=0 \Rightarrow v=w ; \\
d_{b_{2}} . & d_{b}(w, v)=d_{b}(v, w) ; \\
d_{b_{3}} . & d_{b}(u, w) \leq s\left[d_{b}(u, v)+d_{b}(v, w)\right] \text { for all } u, v, w \in S .
\end{array}
$$

In this case, $\left(\mathrm{S}, d_{\mathrm{b}}, \mathrm{S}\right)$ is a dislocated $\mathrm{b}$-metric space (shortly $d_{\mathrm{b}}-\mathrm{MS}$ ).

We mention that, when $\mathrm{s}=1$, a $d_{\mathrm{b}}$-MS becomes a $d_{l}$-MS.

Definition 7 ([36]) A sequence $\left\{\mathrm{v}_{n}\right\}$ on a $d_{\mathrm{b}}-\mathrm{MS}\left(\mathrm{S}, d_{\mathrm{b}}, \mathrm{s}\right)$ is said to be:

- $d_{\mathrm{b}}$-convergent to a point $\mathrm{v} \in \mathrm{S} \Leftrightarrow \lim _{n \rightarrow \infty} d_{\mathrm{b}}\left(\mathrm{v}_{n}, \mathrm{v}\right)=0$;

- $d_{\mathrm{b}}$-Cauchy if and only if $\lim _{n, p \rightarrow \infty} d_{\mathrm{b}}\left(\mathrm{v}_{n}, \mathrm{v}_{p}\right)$ exists and tends to be finite.

Proposition 8 ([36]) In a $d_{b}$-MS the limit of a convergent sequence is unique.

Proposition 9 ([36]) In a $d_{b}$-MS every convergent sequence is $d_{b}$-Cauchy.

In case every $d_{b}$-Cauchy sequence is $d_{b}$-convergent, we say that the space $\left(\mathrm{S}, d_{\mathrm{b}}, \mathrm{s}\right)$ is a complete $d_{b}$-MS. The next lemma will be useful in the sequel.

Lemma 10 Let a $d_{\mathrm{b}}-\mathrm{MS}\left(\mathrm{S}, d_{\mathrm{b}}, \mathrm{S} \geq 1\right)$, a mapping $z: \mathrm{S} \rightarrow \mathrm{S}$, and $\mathrm{v}_{0}$ be arbitrary, but fixed point in $\mathrm{S}$. If there exists $\mathcal{C} \in[0,1)$ such that

$$
d_{b}\left(z^{n} v_{0}, z^{n+1} v_{0}\right) \leq \mathcal{C} d_{b}\left(z^{n-1} v_{0}, z^{n} v_{0}\right)
$$

for every $n \in \mathbb{N}$, then the sequence $\left\{z^{n} v_{0}\right\}$ is a $d_{b}$-Cauchy sequence.

Proof Let $\mathrm{v}_{0}$ be an arbitrary point in $\mathrm{S}$ and the sequence $\left\{\mathrm{v}_{n}\right\}$ with

$$
\mathrm{v}_{1}=Z \mathrm{v}_{0}, \quad \mathrm{v}_{2}=Z \mathrm{v}_{1}=z^{2} \mathrm{v}_{0}, \ldots \mathrm{v}_{n+1}=Z \mathrm{v}_{n}=Z^{n} \mathrm{v}_{0}
$$

for $n \in \mathbb{N} \cup\{0\}$. Thus, by (3), we have

$$
d_{\mathrm{b}}\left(\mathrm{v}_{n}, \mathrm{v}_{n+1}\right) \leq \mathcal{C} d_{\mathrm{b}}\left(\mathrm{v}_{n-1}, \mathrm{v}_{n}\right) \leq \mathcal{C}^{2} d_{\mathrm{b}}\left(\mathrm{v}_{n-2}, \mathrm{v}_{n-1}\right) \leq \cdots \leq \mathcal{C}^{n} d_{\mathrm{b}}\left(\mathrm{v}_{0}, \mathrm{v}_{1}\right)
$$

We split the proof in two cases, namely $s=1$ and $s>1$.

1. For $\mathrm{s}=1$, $d_{\mathrm{b}}$ becomes a dislocated metric and by $d_{l_{3}}$, for $n<p$, we have

$$
\begin{aligned}
d_{\mathrm{b}}\left(\mathrm{v}_{n}, \mathrm{v}_{p}\right) & \leq d_{\mathrm{b}}\left(\mathrm{v}_{n}, \mathrm{v}_{n+1}\right)+d_{\mathrm{b}}\left(\mathrm{v}_{n+1}, \mathrm{v}_{n+2}\right)+\cdots+d_{\mathrm{b}}\left(\mathrm{v}_{p-1}, \mathrm{v}_{p}\right) \\
& \leq \mathcal{C}^{n} d_{\mathrm{b}}\left(\mathrm{v}_{0}, \mathrm{v}_{1}\right)+\mathcal{C}^{n+1} d_{\mathrm{b}}\left(\mathrm{v}_{0}, \mathrm{v}_{1}\right)+\cdots+\mathcal{C}^{p-1} d_{\mathrm{b}}\left(\mathrm{v}_{0}, \mathrm{v}_{1}\right) \\
& =\mathcal{C}^{n} \frac{1-\mathcal{C}^{p-n}}{1-\mathcal{C}} d_{\mathrm{b}}\left(\mathrm{v}_{0}, \mathrm{v}_{1}\right) \rightarrow 0, \quad \text { as } n, p \rightarrow \infty
\end{aligned}
$$

Therefore, $\lim _{n, p \rightarrow \infty} d_{b}\left(v_{n}, v_{p}\right)=0$, that is, the sequence $\left\{z^{n} v_{0}\right\}$ is Cauchy.

2. For $\mathrm{s}>1$, we distinguish two sub-cases: 
(a) If $\mathcal{C} \in\left[0, \frac{1}{s}\right)$, by $\left(d_{b_{3}}\right)$ and taking into account (4), we get

$$
\begin{aligned}
d_{\mathrm{b}}\left(\mathrm{v}_{n}, \mathrm{v}_{p}\right) & \leq \mathrm{s}\left[d_{\mathrm{b}}\left(\mathrm{v}_{n}, \mathrm{v}_{n+1}\right)+d_{\mathrm{b}}\left(\mathrm{v}_{n+1}, \mathrm{v}_{p}\right)\right] \\
& \leq \mathrm{s} d_{\mathrm{b}}\left(\mathrm{v}_{n}, \mathrm{v}_{n+1}\right)+\mathrm{s}^{2} d_{\mathrm{b}}\left(\mathrm{v}_{n+1}, \mathrm{v}_{n+2}\right)+\cdots+\mathrm{s}^{p-n-1} d_{\mathrm{b}}\left(\mathrm{v}_{p-1}, \mathrm{v}_{p}\right) \\
& \leq \mathrm{s} \mathcal{C}^{n} d_{\mathrm{b}}\left(\mathrm{v}_{0}, \mathrm{v}_{1}\right)+\mathrm{s}^{2} \mathcal{C}^{n+1} d_{\mathrm{b}}\left(\mathrm{v}_{0}, \mathrm{v}_{1}\right)+\cdots+\mathrm{s}^{p-n-1} \mathcal{C}^{p-1} d_{\mathrm{b}}\left(\mathrm{v}_{0}, \mathrm{v}_{1}\right) \\
& =s \mathcal{C}^{n}\left(\frac{1-(\mathrm{s} \mathcal{C})^{p}}{1-\mathrm{s} \mathcal{C}}+\frac{(\mathrm{s} \mathcal{C})^{p-n-1}}{\mathrm{~s}}\right) d_{\mathrm{b}}\left(\mathrm{v}_{0}, \mathrm{v}_{1}\right) \rightarrow 0, \quad \text { as } n, p \rightarrow \infty,
\end{aligned}
$$

that is, $\left\{z^{n} v_{0}\right\}$ is $d_{\mathrm{b}}$-Cauchy.

(b) If $\mathcal{C} \in\left[\frac{1}{s}, 1\right)$, then $\mathcal{C}^{n} \rightarrow 0$, and we can find $l \in \mathbb{N}$ such that $\mathcal{C}^{n}<\frac{1}{s}$. Therefore, by (a), the sequence $\left\{Z^{l+n} v_{0}\right\} n \geq 0$ is $d_{b}$-Cauchy. But we have

$$
\left\{\mathrm{v}_{n}\right\}=\left\{\mathrm{v}_{0}, \mathrm{v}_{1}, \ldots, \mathrm{v}_{l-1}\right\} \cup\left\{\mathrm{v}_{l}, \mathrm{v}_{l+1}, \ldots, \mathrm{v}_{l+n}, \ldots\right\}
$$

and then the sequence $\left\{z^{n} v_{0}\right\} n \geq 0$ is $d_{b}$-Cauchy.

\section{Main results}

Henceforth, we use the following notations:

$$
\Theta=\{\Phi, \Psi:(0, \infty) \rightarrow \mathbb{R} \mid \Phi(\theta)<\Psi(\theta) \text { for every } \theta \in(0,+\infty)\}
$$

and, respectively,

$$
\begin{aligned}
& \mathcal{F}_{\mathrm{S}}(Z)=\{\mathrm{x} \in \mathrm{S} \mid Z \mathrm{x}=\mathrm{x}\} \\
& \mathcal{F}_{\mathrm{S}}(\mathcal{U})=\{\mathrm{x} \in \mathrm{S} \mid \mathcal{U} \mathrm{x}=\mathrm{x}\} ; \\
& \mathcal{F}_{\mathrm{S}}(Z, \mathcal{U})=\{\mathrm{x} \in \mathrm{S} \mid Z \mathrm{x}=\mathrm{x}=\mathcal{U} \mathrm{x}\}
\end{aligned}
$$

Let the functions $R_{1}, R_{2}: \mathrm{S} \times \mathrm{S} \rightarrow[0, \infty)$ be defined by

$$
\begin{aligned}
R_{1}(\mathrm{v}, \mathrm{w})= & c_{1} d_{\mathrm{b}}(\mathrm{v}, \mathrm{w})+c_{2} d_{\mathrm{b}}(\mathrm{v}, Z \mathrm{v})+c_{3} d_{\mathrm{b}}(\mathrm{w}, \mathcal{U} \mathrm{w}) \\
& +c_{4} \frac{d_{\mathrm{b}}(\mathrm{w}, \mathcal{U} \mathrm{w}) d_{\mathrm{b}}(\mathrm{v}, Z \mathrm{v})}{d_{\mathrm{b}}(\mathrm{v}, \mathrm{w})} \quad \text { for all } \mathrm{v}, \mathrm{w} \in \mathrm{S}, \mathrm{v} \neq \mathrm{w} ; \\
R_{2}(\mathrm{v}, \mathrm{w})= & c_{1} d_{\mathrm{b}}(\mathrm{v}, \mathrm{w})+c_{2} d_{\mathrm{b}}(\mathrm{v}, Z \mathrm{v})+c_{3} d_{\mathrm{b}}(\mathrm{w}, \mathcal{U} \mathrm{w})+c_{4} \frac{d_{\mathrm{b}}(\mathrm{w}, \mathcal{U} \mathrm{w}) d_{\mathrm{b}}(\mathrm{v}, Z \mathrm{v})}{1+d_{\mathrm{b}}(\mathrm{v}, \mathrm{w})} \\
& +c_{5} \frac{d_{\mathrm{b}}(\mathrm{w}, \mathcal{U} \mathrm{w})\left(1+d_{\mathrm{b}}(\mathrm{v}, Z \mathrm{v})\right)}{1+d_{\mathrm{b}}(\mathrm{v}, \mathrm{w})} \quad \text { for all } \mathrm{v}, \mathrm{w} \in \mathrm{S},
\end{aligned}
$$

where $c_{1}, c_{2}, c_{3}, c_{4}, c_{5}$ are nonnegative real numbers.

Theorem 11 Let $\left(\mathrm{S}, d_{\mathrm{b}}, \mathrm{s}\right)$ be a complete $d_{\mathrm{b}}-\mathrm{ms}, \Psi, \Phi \in \Theta$, a number $\alpha \in[1, \infty)$, and two continuous mappings $Z, \mathcal{Z}: \mathrm{S} \rightarrow \mathrm{S}$ such that, for every distinct $\mathrm{v}, \mathrm{w} \in \mathrm{S}$ with $d_{\mathrm{b}}(Z \mathrm{v}, \mathcal{u} \mathrm{w})>0$, the following inequality

$$
\Psi\left(\mathrm{s}^{\alpha} d_{\mathrm{b}}(Z \mathrm{v}, \mathcal{U} \mathrm{w})\right) \leq \Phi\left(R_{1}(\mathrm{v}, \mathrm{w})\right)
$$

holds. Assume that: 
$\left(\beta_{1}\right) c_{1}+c_{2}+c_{3}+c_{4}<\mathrm{s}^{\alpha}$ and $c_{1}>0$

$\left(\beta_{2}\right) \Psi$ is nondecreasing.

Then $\mathcal{F}_{\mathrm{S}}\left(Z, \mathcal{Z}, \neq \emptyset\right.$. Moreover, if $c_{1}+2 c_{2}+2 c_{3}+4 c_{4} \leq \mathrm{s}^{\alpha}$, then the set $\mathcal{F}_{\mathrm{S}}(Z, \mathcal{Z}$, has exactly one element.

Proof For an arbitrary (but fixed) point $\mathrm{v}_{0} \in \mathrm{S}$, let $\left\{\mathrm{v}_{n}\right\}$ be the sequence defined as follows:

$$
\mathrm{v}_{1}=Z \mathrm{v}_{0}, \quad \mathrm{v}_{2}=\mathcal{U} \mathrm{v}_{1}, \ldots, \mathrm{v}_{2 n+1}=Z \mathrm{v}_{2 n}, \quad \mathrm{v}_{2 n+2}=\mathcal{U} \mathrm{v}_{2 n+1}, \ldots
$$

for all $n \in \mathbb{N}_{0}$. First of all, we claim that $\mathrm{v}_{n} \neq \mathrm{v}_{n+1}$ for any $n \in \mathbb{N}_{0}$. Indeed, if we can find $l_{0} \in \mathbb{N}$ such that $\mathrm{v}_{l_{0}}=\mathrm{v}_{l_{0}+1}=\mathrm{v}_{l_{0}+2}=\mathrm{x}$, then $\mathrm{x} \in \mathcal{F}_{\mathrm{S}}(Z, \mathcal{L})$.

Under this assumption, $d\left(Z \mathbf{v}_{2 n}, U \mathbf{v}_{2 n+1}\right)=d\left(\mathbf{v}_{2 n+1}, \mathrm{v}_{2 n+2}\right)>0$ and letting $\mathrm{v}=\mathrm{v}_{2 n}$ and $\mathrm{w}=$ $\vee_{2 n+1}$ in (5), because the functions $\Psi, \Phi$ belong to $\Theta$, we have

$$
\begin{aligned}
\Psi\left(\mathrm{s}^{\alpha} d_{\mathrm{b}}\left(\mathrm{v}_{2 n+1}, \mathrm{v}_{2 n+2}\right)\right) & =\Psi\left(\mathrm{s}^{\alpha} d_{\mathrm{b}}\left(Z \mathrm{v}_{2 n}, \mathcal{U} \mathrm{v}_{2 n+1}\right)\right) \leq \Phi\left(R_{1}\left(\mathrm{v}_{2 n}, \mathrm{v}_{2 n+1}\right)\right) \\
& \leq \Phi\left(\begin{array}{c}
c_{1} d_{\mathrm{b}}\left(\mathrm{v}_{2 n}, \mathrm{v}_{2 n+1}\right)+c_{2} d_{\mathrm{b}}\left(\mathrm{v}_{2 n}, Z \mathrm{v}_{2 n}\right)+c_{3} d_{\mathrm{b}}\left(\mathrm{v}_{2 n+1}, \mathcal{U} \mathrm{v}_{2 n+1}\right)+ \\
+c_{4} \frac{d_{\mathrm{b}}\left(\mathrm{v}_{2 n+1}, \mathcal{u} \mathrm{v}_{2 n+1}\right) d_{\mathrm{b}}\left(\mathrm{v}_{2 n}, z \mathrm{v}_{2 n}\right)}{d_{\mathrm{b}}\left(\mathrm{v}_{2 n}, \mathrm{v}_{2 n+1}\right)}
\end{array}\right) \\
& =\Phi\left(\begin{array}{c}
c_{1} d_{\mathrm{b}}\left(\mathrm{v}_{2 n}, \mathrm{v}_{2 n+1}\right)+c_{2} d_{\mathrm{b}}\left(\mathrm{v}_{2 n}, \mathrm{v}_{2 n+1}\right)+c_{3} d_{\mathrm{b}}\left(\mathrm{v}_{2 n+1}, \mathrm{v}_{2 n+2}\right)+ \\
+c_{4} \frac{d_{\mathrm{b}}\left(\mathrm{v}_{2 n+1}, \mathrm{v}_{2 n+2}\right) d_{\mathrm{b}}\left(\mathrm{v}_{2 n}, \mathrm{v}_{2 n+1}\right)}{d_{\mathrm{b}}\left(\mathrm{v}_{2 n}, \mathrm{v}_{2 n+1}\right)}
\end{array}\right) \\
& =\Phi\left(\left(c_{1}+c_{2}\right) d_{\mathrm{b}}\left(\mathrm{v}_{2 n}, \mathrm{v}_{2 n+1}\right)+\left(c_{3}+c_{4}\right) d_{\mathrm{b}}\left(\mathrm{v}_{2 n+1}, \mathrm{v}_{2 n+2}\right)\right) \\
& <\Psi\left(\left(c_{1}+c_{2}\right) d_{\mathrm{b}}\left(\mathrm{v}_{2 n}, \mathrm{v}_{2 n+1}\right)+\left(c_{3}+c_{4}\right) d_{\mathrm{b}}\left(\mathrm{v}_{2 n+1}, \mathrm{v}_{2 n+2}\right)\right) .
\end{aligned}
$$

Taking $\left(\beta_{1}\right)$ into account, we get

$$
\mathrm{s}^{\alpha} d_{\mathrm{b}}\left(\mathrm{v}_{2 n+1}, \mathrm{v}_{2 n+2}\right)<\left(c_{1}+c_{2}\right) d_{\mathrm{b}}\left(\mathrm{v}_{2 n}, \mathrm{v}_{2 n+1}\right)+\left(c_{3}+c_{4}\right) d_{\mathrm{b}}\left(\mathrm{v}_{2 n+1}, \mathrm{v}_{2 n+2}\right)
$$

or

$$
d_{\mathrm{b}}\left(\mathrm{v}_{2 n+1}, \mathrm{v}_{2 n+2}\right)<\frac{c_{1}+c_{2}}{\mathrm{~s}^{r}-c_{3}-c_{4}} d_{\mathrm{b}}\left(\mathrm{v}_{2 n}, \mathrm{v}_{2 n+1}\right)=\mathcal{K} \cdot d_{\mathrm{b}}\left(\mathrm{v}_{2 n}, \mathrm{v}_{2 n+1}\right),
$$

where $\mathcal{K}=\frac{c_{1}+c_{2}}{s^{r}-c_{3}-c_{4}}<1$, holds due to the first assumption in $\left(\beta_{1}\right)$.

In the same way, replacing in (5) $v$ with $v_{2 n-1}$ and $w$ with $v_{2 n}$, and keeping in mind $\left(d_{\mathrm{b}_{2}}\right)$, we have

$$
\begin{aligned}
\Psi\left(\mathrm{s}^{\alpha} d_{\mathrm{b}}\left(\mathrm{v}_{2 n}, \mathrm{v}_{2 n+1}\right)\right) & =\Psi\left(\mathrm{s}^{\alpha} d_{\mathrm{b}}\left(\mathcal{U} \mathrm{v}_{2 n-1}, z \mathrm{v}_{2 n}\right)\right) \leq \Phi\left(R_{1}\left(\mathrm{v}_{2 n-1}, \mathrm{v}_{2 n}\right)\right) \\
& =\Phi\left(\left(c_{1}+c_{2}\right) d_{\mathrm{b}}\left(\mathrm{v}_{2 n-1}, \mathrm{v}_{2 n}\right)+\left(c_{3}+c_{4}\right) d_{\mathrm{b}}\left(\mathrm{v}_{2 n}, \mathrm{v}_{2 n+1}\right)\right) \\
& <\Psi\left(\left(c_{1}+c_{2}\right) d_{\mathrm{b}}\left(\mathrm{v}_{2 n-1}, \mathrm{v}_{2 n}\right)+\left(c_{3}+c_{4}\right) d_{\mathrm{b}}\left(\mathrm{v}_{2 n}, \mathrm{v}_{2 n+1}\right)\right)
\end{aligned}
$$

which leads us to

$$
d_{\mathrm{b}}\left(\mathrm{v}_{2 n}, \mathrm{v}_{2 n+1}\right)<\frac{c_{1}+c_{2}}{\mathrm{~s}^{\alpha}-c_{3}-c_{4}} d_{\mathrm{b}}\left(\mathrm{v}_{2 n-1}, \mathrm{v}_{2 n}\right)=\mathcal{K} \cdot d_{\mathrm{b}}\left(\mathrm{v}_{2 n-1}, \mathrm{v}_{2 n}\right)
$$


Consequently, (7) and (9) show us that

$$
d_{\mathrm{b}}\left(\mathrm{v}_{n}, \mathrm{v}_{n+1}\right)<\mathcal{K} \cdot d_{\mathrm{b}}\left(\mathrm{v}_{n-1}, \mathrm{v}_{n}\right)
$$

for any $n \in \mathbb{N}$, where $\mathcal{K} \in(0,1)$. By Lemma 10 it follows that $\left\{\mathrm{v}_{n}\right\}$ is a Cauchy sequence. Thus, $\lim _{n, p \rightarrow \infty} d_{b}\left(\mathrm{v}_{n}, \mathrm{v}_{p}\right)$ exists and is finite. Moreover, since the $d_{\mathrm{b}}$ - $\mathrm{ms}$ is complete, we get that there exists $\mathrm{x} \in \mathrm{S}$ such that $\lim _{n \rightarrow \infty} \mathrm{v}_{n}=\mathrm{x}$ and

$$
\lim _{n \rightarrow \infty} d_{\mathrm{b}}\left(\mathrm{v}_{n}, \mathrm{x}\right)=\lim _{n, p \rightarrow \infty} d_{\mathrm{b}}\left(\mathrm{v}_{n}, \mathrm{v}_{p}\right)=0
$$

Since the mappings $Z$ and $\mathcal{U}$ are supposed to be continuous, we have

$$
\begin{aligned}
Z \mathrm{x} & =z\left(\lim _{n \rightarrow \infty} \mathrm{v}_{2 n}\right)=\lim _{n \rightarrow \infty} z \mathrm{v}_{2 n}=\lim _{n \rightarrow \infty} \mathrm{v}_{2 n+1}=\mathrm{x} \\
& =\lim _{n \rightarrow \infty} \mathrm{v}_{2 n+2}=\lim _{n \rightarrow \infty} \mathcal{U} \mathrm{v}_{2 n+1} \\
& =u\left(\lim _{n \rightarrow \infty} \mathrm{v}_{2 n+1}\right)=\mathcal{U} \mathrm{x},
\end{aligned}
$$

that is, $\mathcal{F}_{\mathrm{S}}(\mathcal{Z}, \mathcal{U}) \neq \emptyset$. If we suppose that there exist $\mathrm{x}, \mathrm{y} \in \mathcal{F}_{\mathrm{S}}(Z, \mathcal{U})$ such that $\mathrm{x} \neq \mathrm{y}$, by (5) and since $\Psi, \Phi \in \Theta$, we have

$$
\Psi\left(\mathrm{s}^{\alpha} d_{\mathrm{b}}(\mathrm{x}, \mathrm{y})\right)=\Psi\left(\mathrm{s}^{\alpha} d_{\mathrm{b}}(z \mathrm{x}, \tau \mathrm{y} \mathrm{y})\right) \leq \Phi\left(R_{1}(\mathrm{x}, \mathrm{y})\right)<\Psi\left(R_{1}(\mathrm{x}, \mathrm{y})\right)
$$

where

$$
\begin{aligned}
R_{1}(\mathrm{x}, \mathrm{y}) & =c_{1} d_{\mathrm{b}}(\mathrm{x}, \mathrm{y})+c_{2} d_{\mathrm{b}}(\mathrm{x}, z \mathrm{x})+c_{3} d_{\mathrm{b}}(\mathrm{y}, \tau \mathrm{y})+c_{4} \frac{d_{\mathrm{b}}(\mathrm{y}, \tau \mathrm{y}) d_{\mathrm{b}}(\mathrm{x}, z \mathrm{x})}{d_{\mathrm{b}}(\mathrm{x}, \mathrm{y})} \\
& =c_{1} d_{\mathrm{b}}(\mathrm{x}, \mathrm{y})+c_{2} d_{\mathrm{b}}(\mathrm{x}, \mathrm{x})+c_{3} d_{\mathrm{b}}(\mathrm{y}, \mathrm{y})+c_{4} \frac{d_{\mathrm{b}}(\mathrm{y}, \mathrm{y}) d_{\mathrm{b}}(\mathrm{x}, \mathrm{x})}{d_{\mathrm{b}}(\mathrm{x}, \mathrm{y})} .
\end{aligned}
$$

However, applying $\left(d_{\mathrm{b}_{3}}\right)$ and taking into account $\left(d_{\mathrm{b}_{2}}\right)$,

$$
\begin{aligned}
R_{1}(\mathrm{x}, \mathrm{y}) & \leq c_{1} d_{\mathrm{b}}(\mathrm{x}, \mathrm{y})+2 c_{2} d_{\mathrm{b}}(\mathrm{x}, \mathrm{y})+2 c_{3} d_{\mathrm{b}}(\mathrm{x}, \mathrm{y})+4 c_{4} \frac{d_{\mathrm{b}}^{2}(\mathrm{x}, \mathrm{y})}{d_{\mathrm{b}}(\mathrm{x}, \mathrm{y})} \\
& =\left(c_{1}+2 c_{2}+2 c_{3}+4 c_{4}\right) d_{\mathrm{b}}(\mathrm{x}, \mathrm{y}) .
\end{aligned}
$$

Moreover, by $\left(\beta_{2}\right)$ we get

$$
\mathrm{s}^{\alpha} d_{\mathrm{b}}(\mathrm{x}, \mathrm{y})<\left(c_{1}+2 c_{2}+2 c_{3}+4 c_{4}\right) d_{\mathrm{b}}(\mathrm{x}, \mathrm{y}) \leq \mathrm{s}^{\alpha} d_{\mathrm{b}}(\mathrm{x}, \mathrm{y})
$$

which is a contradiction. Therefore, $d_{\mathrm{b}}(\mathrm{x}, \mathrm{y})=0$ and from $\left(d_{\mathrm{b}_{1}}\right)$ it follows that $\mathrm{x}=\mathrm{y}$, that is, the set $\mathcal{F}_{\mathrm{S}}(Z, \mathcal{U})$ has exactly one element.

Corollary 12 Let $\left(\mathrm{S}, d_{\mathrm{b}}, \mathrm{s}\right)$ be a complete $d_{\mathrm{b}}-\mathrm{ms}, \Psi, \Phi \in \Theta$, a number $\alpha \in[1, \infty)$, and a continuous mapping $\mathrm{Z}: \mathrm{S} \rightarrow \mathrm{S}$ such that, for every distinct $\mathrm{v}, \mathrm{w} \in \mathrm{S}$ with $d_{\mathrm{b}}(\mathrm{zv}, Z \mathrm{w})>0$, the following inequality

$$
\Psi\left(\mathrm{s}^{\alpha} d_{\mathrm{b}}(z \mathrm{v}, z \mathrm{w})\right) \leq \Phi\left(R_{1}^{*}(\mathrm{v}, \mathrm{w})\right)
$$


holds, where for $c_{1}, c_{2}, c_{3}, c_{4}$ nonnegative real numbers,

$$
R_{1}^{*}(\mathrm{v}, \mathrm{w})=c_{1} d_{\mathrm{b}}(\mathrm{v}, \mathrm{w})+c_{2} d_{\mathrm{b}}(\mathrm{v}, Z \mathrm{v})+c_{3} d_{\mathrm{b}}(\mathrm{w}, Z \mathrm{w})+c_{4} \frac{d_{\mathrm{b}}(\mathrm{w}, Z \mathrm{w}) d_{\mathrm{b}}(\mathrm{v}, Z \mathrm{v})}{d_{\mathrm{b}}(\mathrm{v}, \mathrm{w})}
$$

for all $\mathrm{v}, \mathrm{w} \in \mathrm{S}, \mathrm{v} \neq \mathrm{w}$. Assume that:

$\left(\beta_{1}\right) c_{1}+c_{2}+c_{3}+c_{4}<\mathrm{s}^{\alpha}$ and $c_{1}>0$

$\left(\beta_{2}\right) \Psi$ is nondecreasing.

Then $\mathcal{F}_{\mathrm{S}}(Z) \neq \emptyset$. Moreover, if $c_{1} \leq 1$, then the set $\mathcal{F}_{\mathrm{S}}(Z)$ has exactly one element.

Proof Let $z=\mathcal{U}$ in Theorem 11.

Theorem 13 Let $\left(\mathrm{S}, d_{\mathrm{b}}, \mathrm{s}\right)$ be a complete $d_{\mathrm{b}}-\mathrm{ms}, \Psi, \Phi \in \Theta$, a number $\alpha \in[1, \infty)$, and two mappings $z, \mathcal{u}: \mathrm{S} \rightarrow \mathrm{S}$ such that, for every distinct $\mathrm{v}, \mathrm{w} \in \mathrm{S}$ with $\alpha_{\mathrm{b}}(z \mathrm{v}, \mathcal{u} \mathrm{w})>0$, the following inequality

$$
\Psi\left(\mathrm{s}^{\alpha} d_{\mathrm{b}}(Z \mathrm{v}, \mathcal{U} \mathrm{w})\right) \leq \Phi\left(R_{2}(\mathrm{v}, \mathrm{w})\right)
$$

holds. Assume that:

$\left(\beta_{1}\right) c_{1}+c_{2}+c_{3}+c_{4}+c_{5}<s^{\alpha}, c_{1}>0, c_{2} \leq 1, c_{3}+c_{5} \leq 1$

$\left(\beta_{2}\right) \Psi$ is nondecreasing.

Then $\mathcal{F}_{\mathcal{S}}(\mathcal{Z}, \mathcal{U}) \neq \emptyset$. Moreover, if $c_{1} \leq 1$, then the set $\mathcal{F}_{\mathcal{S}}(Z, \mathcal{Z})$ has exactly one element.

Proof Let $\mathrm{v}_{0} \in \mathrm{S}$ be a chosen point and $\left\{\mathrm{v}_{n}\right\}$ be the sequence defined by (6) in the proof of Theorem 11 . Thus, following the same arguments, we can assume that $d_{b}\left(z v_{2 n}, \mathcal{U} v_{2 n+1}\right)>0$ and from (13) we get

$$
\begin{aligned}
& \Psi\left(\mathrm{s}^{\alpha} d_{\mathrm{b}}\left(\mathrm{v}_{2 n+1}, \mathrm{v}_{2 n+2}\right)\right)=\Psi\left(\mathrm{s}^{\alpha} d_{\mathrm{b}}\left(z \mathrm{v}_{2 n}, \mathcal{u} \mathrm{v}_{2 n+1}\right)\right) \leq \Phi\left(R_{2}\left(\mathrm{v}_{2 n}, \mathrm{v}_{2 n+1}\right)\right) \\
& =\Phi\left(\begin{array}{c}
c_{1} d_{b}\left(v_{2 n}, v_{2 n+1}\right)+c_{2} d_{b}\left(v_{2 n}, z v_{2 n}\right)+c_{3} d_{b}\left(v_{2 n+1}, \tau v_{2 n+1}\right)+ \\
+c_{4} \frac{d_{b}\left(v_{2 n+1}, v_{2 n+1}\right) d_{b}\left(v_{2 n}, z v_{2 n}\right)}{1+d_{b}\left(v_{2 n}, v_{2 n+1}\right)}+c_{5} \frac{d_{b}\left(v_{2 n+1}, q v_{2 n+1}\right)\left(1+d_{b}\left(v_{2 n}, z v_{2 n}\right)\right]}{1+d_{b}\left(v_{2 n}, v_{2 n+1}\right)}
\end{array}\right) \\
& <\Psi\left(\begin{array}{c}
\left(c_{1} d_{b}\left(\mathrm{v}_{2 n}, \mathrm{v}_{2 n+1}\right)+c_{2} d_{b}\left(\mathrm{v}_{2 n}, \mathrm{v}_{2 n+1}\right)+c_{3} d_{b}\left(\mathrm{v}_{2 n+1}, \mathrm{v}_{2 n+2}\right)+\right. \\
+c_{4} \frac{d_{b}\left(\mathrm{v}_{2 n+1}, \mathrm{v}_{2 n+2}\right) d_{d}\left(\mathrm{v}_{2 n}, \mathrm{v}_{2 n+1}\right)}{1+d_{b}\left(\mathrm{v}_{2 n}, \mathrm{v}_{2 n+1}\right)}+c_{5} \frac{d_{b}\left(\mathrm{v}_{2 n+1}, \mathrm{v}_{2 n+2}\right)\left(1+1+d_{b}\left(\mathrm{v}_{2 n}, \mathrm{v}_{2 n+1}\right)\right]}{1+d_{b}\left(\mathrm{v}_{2 n}, \mathrm{v}_{2 n+1}\right)}
\end{array}\right) \\
& \leq \Psi\left(\left(c_{1}+c_{2}\right) d_{\mathrm{b}}\left(\mathrm{v}_{2 n}, \mathrm{v}_{2 n+1}\right)+\left(c_{3}+c_{4}+c_{5}\right) d_{\mathrm{b}}\left(\mathrm{v}_{2 n+1}, \mathrm{v}_{2 n+2}\right)\right) \text {. }
\end{aligned}
$$

Since by $\left(\beta_{2}\right) \Psi$ is nondecreasing, we deduce that

$$
0<\mathrm{s}^{\alpha} d_{\mathrm{b}}\left(\mathrm{v}_{2 n+1}, \mathrm{v}_{2 n+2}\right)<\left(c_{1}+c_{2}\right) d_{\mathrm{b}}\left(\mathrm{v}_{2 n}, \mathrm{v}_{2 n+1}\right)+\left(c_{3}+c_{4}+c_{5}\right) d_{\mathrm{b}}\left(\mathrm{v}_{2 n+1}, \mathrm{v}_{2 n+2}\right),
$$

which is equivalent to

$$
0<d_{\mathrm{b}}\left(\mathrm{v}_{2 n+1}, \mathrm{v}_{2 n+2}\right)<\mathcal{C} d_{\mathrm{b}}\left(\mathrm{v}_{2 n}, \mathrm{v}_{2 n+1}\right)
$$

where $\mathcal{C}=\frac{c_{1}+c_{2}}{s^{\alpha}-c_{3}-c_{4}-c_{5}}<1$ by $\left(\beta_{1}\right)$. Similarly, taking $\mathrm{v}=\mathrm{v}_{2 n}$ and $\mathrm{w}=\mathrm{v}_{2 n-1}$ in (5) and keeping in mind $\left(d_{b_{2}}\right)$, we get

$$
0<d_{\mathrm{b}}\left(\mathrm{v}_{2 n}, \mathrm{v}_{2 n+1}\right)<\mathcal{C}_{1} d_{\mathrm{b}}\left(\mathrm{v}_{2 n+1}, \mathrm{v}_{2 n+2}\right) .
$$


However, from relations (14), (15), together with Lemma 10, we find that $\left\{\mathrm{v}_{n}\right\}$ is a Cauchy sequence in a complete $d_{b}$-ms. Therefore, there exists $x \in S$ such that

$$
\lim _{n, p \rightarrow \infty} d_{b}\left(\mathrm{v}_{n}, \mathrm{v}_{p}\right)=0=\lim _{n \rightarrow \infty} d_{\mathrm{b}}\left(\mathrm{v}_{n}, \mathrm{x}\right)
$$

Without loss of generality, we can suppose that $x \neq v_{n}$ for any $n \in \mathbb{N}$. Supposing that $x \neq Z x$, by (5), we have

$$
\Psi\left(\mathrm{s}^{\alpha} d_{\mathrm{b}}\left(Z \mathrm{x}, \mathcal{U} \mathrm{v}_{2 m-1}\right)\right) \leq \Phi\left(R_{2}\left(\mathrm{x}, \mathrm{v}_{2 m-1}\right)\right)<\Psi\left(R_{2}\left(\mathrm{x}, \mathrm{v}_{2 m-1}\right)\right)
$$

or, taking $\left(\beta_{2}\right)$ into account,

$$
\mathrm{s}^{\alpha} d_{\mathrm{b}}\left(Z \mathrm{x}, \mathcal{U} \mathrm{v}_{2 m-1}\right)<R_{2}\left(\mathrm{x}, \mathrm{v}_{2 m-1}\right)
$$

However, since

$$
\begin{aligned}
R_{2}\left(\mathrm{x}, \mathrm{v}_{2 m-1}\right)= & c_{1} d_{\mathrm{b}}\left(\mathrm{x}, \mathrm{v}_{2 m-1}\right)+c_{2} d_{\mathrm{b}}(\mathrm{x}, Z \mathrm{x})+c_{3} d_{\mathrm{b}}\left(\mathrm{v}_{2 m-1}, \mathcal{U} \mathrm{v}_{2 m-1}\right) \\
& +c_{4} \frac{d_{\mathrm{b}}\left(\mathrm{v}_{2 m-1}, \mathcal{U} \mathrm{v}_{2 m-1}\right) d_{\mathrm{b}}(\mathrm{x}, Z \mathrm{x})}{1+d_{\mathrm{b}}\left(\mathrm{x}, \mathrm{v}_{2 m}\right)}+c_{5} \frac{d_{\mathrm{b}}\left(\mathrm{v}_{2 m-1}, \mathcal{U} \mathrm{v}_{2 m-1}\right)\left[1+d_{\mathrm{b}}(\mathrm{x}, Z \mathrm{x})\right]}{1+d_{\mathrm{b}}\left(\mathrm{x}, \mathrm{v}_{2 m}\right)} \\
= & c_{1} d_{\mathrm{b}}\left(\mathrm{x}, \mathrm{v}_{2 m-1}\right)+c_{2} d_{\mathrm{b}}(\mathrm{x}, Z \mathrm{x})+c_{3} d_{\mathrm{b}}\left(\mathrm{v}_{2 m-1}, \mathrm{v}_{2 m}\right) \\
& +c_{4} \frac{d_{\mathrm{b}}\left(\mathrm{v}_{2 m-1}, \mathrm{v}_{2 m}\right) d_{\mathrm{b}}(\mathrm{x}, Z \mathrm{x})}{1+d_{\mathrm{b}}\left(\mathrm{x}, \mathrm{v}_{2 m-1}\right)}+c_{5} \frac{+d_{\mathrm{b}}\left(\mathrm{v}_{2 m-1}, \mathrm{v}_{2 m}\right)\left[1+d_{\mathrm{b}}(\mathrm{x}, Z \mathrm{x})\right]}{1+d_{\mathrm{b}}\left(\mathrm{x}, \mathrm{v}_{2 m-1}\right)}
\end{aligned}
$$

we obtain

$$
0<\limsup _{m \rightarrow \infty} R_{2}\left(\mathrm{x}, \mathrm{v}_{2 m-1}\right)<\mathcal{c}_{2} d_{\mathrm{b}}(\mathrm{x}, Z \mathrm{x}) .
$$

On the other hand,

$$
\begin{aligned}
d_{\mathrm{b}}(\mathrm{x}, Z \mathrm{x}) & \leq \mathrm{s}\left[d_{\mathrm{b}}\left(\mathrm{x}, \mathrm{v}_{2 m}\right)+d_{\mathrm{b}}\left(\mathrm{v}_{2 m}, Z \mathrm{x}\right)\right] \\
& \leq \mathrm{s} d_{\mathrm{b}}\left(\mathrm{x}, \mathrm{v}_{2 m}\right)+\mathrm{s}^{\alpha} d_{\mathrm{b}}\left(\mathcal{U} \mathrm{v}_{2 m-1}, Z \mathrm{x}\right) \\
& <\mathrm{s} d_{\mathrm{b}}\left(\mathrm{x}, \mathrm{v}_{2 m}\right)+R_{2}\left(\mathrm{x}, \mathrm{v}_{2 m-1}\right)
\end{aligned}
$$

and then

$$
0<\limsup _{m \rightarrow \infty} d_{\mathrm{b}}(\mathrm{x}, Z \mathrm{x})<\limsup _{m \rightarrow \infty} R_{2}\left(\mathrm{x}, \mathrm{v}_{2 m-1}\right)<c_{2} d_{\mathrm{b}}(\mathrm{x}, Z \mathrm{x})
$$

which contradicts our assumption $c_{2} \leq 1$. Thus, we get $d_{b}(x, Z x)=0$, that is, $x=Z x$. Moreover, if we suppose that $\mathrm{x} \notin \mathcal{F}_{\mathrm{S}}(Z, \mathcal{U})$, since $d_{\mathrm{b}}(Z \mathrm{x}, \mathcal{U} \mathrm{x})>0$,

$$
\Psi\left(\mathrm{s} d_{\mathrm{b}}(Z \mathrm{x}, \mathcal{U} \mathrm{x})\right) \leq \Psi\left(\mathrm{s}^{\alpha} d_{\mathrm{b}}(Z \mathrm{x}, \mathcal{U} \mathrm{x})\right) \leq \Phi\left(R_{2}(u, u)\right)<\Psi\left(R_{2}(u, u)\right)
$$

or, keeping in mind $\left(\beta_{2}\right)$

$$
\mathrm{s} d_{\mathrm{b}}(Z \mathrm{x}, \mathcal{U} \mathrm{x})<R_{2}(\mathrm{x}, \mathrm{x})=c_{1} d_{\mathrm{b}}(\mathrm{x}, \mathrm{x})+c_{2} d_{\mathrm{b}}(\mathrm{x}, Z \mathrm{x})+c_{3} d_{\mathrm{b}}(\mathrm{x}, \mathcal{U} \mathrm{x})
$$




$$
\begin{aligned}
& +c_{4} \frac{d_{\mathrm{b}}(\mathrm{x}, \mathcal{U} \mathrm{x}) d_{\mathrm{b}}(\mathrm{x}, Z \mathrm{x})}{1+d_{\mathrm{b}}(\mathrm{x}, \mathrm{x})}+c_{5} \frac{d_{\mathrm{b}}(\mathrm{x}, \mathcal{U} \mathrm{x})\left[1+d_{\mathrm{b}}(\mathrm{x}, Z \mathrm{x})\right]}{1+d_{\mathrm{b}}(\mathrm{x}, \mathrm{x})} \\
& =\left(c_{3}+c_{5}\right) d_{\mathrm{b}}(\mathrm{x}, \mathcal{U} \mathrm{x}) \leq \mathrm{s}\left(c_{3}+c_{5}\right)\left[d_{\mathrm{b}}(\mathrm{x}, Z \mathrm{x})+d_{\mathrm{b}}(z \mathrm{x}, \mathcal{U} \mathrm{x})\right] \\
& =\mathrm{s}\left(c_{3}+c_{5}\right) d_{\mathrm{b}}(Z \mathrm{x}, \mathcal{U} \mathrm{x}) \leq \mathrm{s} d_{\mathrm{b}}(Z \mathrm{x}, \mathcal{U} \mathrm{x}) \text {, }
\end{aligned}
$$

which is a contradiction. Therefore, $d_{\mathrm{b}}(Z \mathrm{x}, \mathcal{U} \mathrm{x})=0$ which implies by $\left(d_{\mathrm{b}_{1}}\right)$ that $\mathrm{x}=\mathcal{Z} \mathrm{x}=\mathcal{U} \mathrm{x}$. That is, $\mathcal{F}_{\mathrm{S}}(Z, \mathcal{U}) \neq \emptyset$.

As a last step, we claim that $\mathrm{x}$ is the unique fixed point of the mappings $z$ and $\mathcal{U}$. Indeed, if we suppose that there exists another point $v \in \mathcal{F}_{\mathrm{S}}(Z, \mathcal{U})$ such that $\mathrm{x} \neq v$, by (13) we have

$$
\begin{aligned}
\Psi\left(d_{\mathrm{b}}(\mathrm{x}, v)\right) & \leq \Psi\left(\mathrm{s}^{\alpha} d_{\mathrm{b}}(\mathrm{x}, v)\right) \leq \Phi\left(R_{2}(\mathrm{x}, v)\right)<\Psi\left(R_{2}(\mathrm{x}, v)\right) \\
& =\Psi\left(c_{1} d_{\mathrm{b}}(\mathrm{x}, v)\right) .
\end{aligned}
$$

Since the function $\Psi$ is supposed to be nondecreasing, it follows that

$$
d_{\mathrm{b}}(\mathrm{x}, v)<c_{1} d_{\mathrm{b}}(\mathrm{x}, v) \leq d_{\mathrm{b}}(\mathrm{x}, v)
$$

which is a contradiction. Therefore, the set $\mathcal{F}_{\mathrm{S}}(Z, \mathcal{Z})$ has exactly one element.

Corollary 14 Let $\left(\mathrm{S}, d_{\mathrm{b}}, \mathrm{s}\right)$ be a complete $d_{\mathrm{b}}-\mathrm{ms}, \Psi, \Phi \in \Theta$, a number $\alpha \in[1, \infty)$, and a mapping $Z: \mathrm{S} \rightarrow \mathrm{S}$ such that, for every $\mathrm{v}, \mathrm{w} \in \mathrm{S}$ with $d_{\mathrm{b}}(Z \mathrm{v}, \mathcal{Z} \mathrm{w})>0$, the following inequality

$$
\Psi\left(\mathrm{s}^{\alpha} d_{\mathrm{b}}(Z \mathrm{v}, \mathcal{U} \mathrm{w})\right) \leq \Phi\left(R_{2}(\mathrm{v}, \mathrm{w})\right)
$$

holds, where for $c_{1}, c_{2}, c_{3}, c_{4}, c_{5}$ nonnegative real numbers,

$$
\begin{aligned}
R_{2}(\mathrm{v}, \mathrm{w})= & c_{1} d_{\mathrm{b}}(\mathrm{v}, \mathrm{w})+c_{2} d_{\mathrm{b}}(\mathrm{v}, Z \mathrm{v})+c_{3} d_{\mathrm{b}}(\mathrm{w}, Z \mathrm{w})+c_{4} \frac{d_{\mathrm{b}}(\mathrm{w}, Z \mathrm{w}) d_{\mathrm{b}}(\mathrm{v}, \mathrm{zv})}{1+d_{\mathrm{b}}(\mathrm{v}, \mathrm{w})} \\
& +c_{5} \frac{d_{\mathrm{b}}(\mathrm{w}, Z \mathrm{w})\left(1+d_{\mathrm{b}}(\mathrm{v}, Z \mathrm{v})\right)}{1+d_{\mathrm{b}}(\mathrm{v}, \mathrm{w})} \text { for all } \mathrm{v}, \mathrm{w} \in \mathrm{S} .
\end{aligned}
$$

\section{Assume that:}

$\left(\beta_{1}\right) c_{1}+c_{2}+c_{3}+c_{4}+c_{5}<\mathrm{s}^{\alpha}, c_{1}>0$, and $c_{3}+c_{5} \leq 1$;

$\left(\beta_{2}\right) \Psi$ is nondecreasing.

Then $\mathcal{F}_{\mathrm{S}}(Z) \neq \emptyset$. Moreover, if $c_{1} \leq 1$, then the set $\mathcal{F}_{\mathrm{S}}(Z)$ has exactly one element.

Proof Let $z=\mathcal{U}$ in Theorem 13.

Example 15 Let the set $\mathcal{U}=\{m, n, p, q\}$ and the function $d_{\mathrm{b}}: \mathrm{S} \times \mathrm{S} \rightarrow[0, \infty)$ be defined by

\begin{tabular}{lllll}
\hline$d_{\mathrm{b}}(\mathrm{v}, \mathrm{w})$ & $m$ & $n$ & $p$ & $q$ \\
\hline$m$ & 0 & 2 & 5 & 7 \\
$n$ & 2 & 6 & 8 & 5 \\
$p$ & 5 & 8 & 0 & 1 \\
$q$ & 7 & 5 & 1 & 0 \\
\hline
\end{tabular}

Obviously, $d_{\mathrm{b}}$ is a $d_{\mathrm{b}}$-metric, with $\mathrm{s}=2$. Let $z, \mathcal{U}: \mathrm{S} \rightarrow \mathrm{S}$ be two mappings, where $z m=$ $z p=Z q=p, Z n=q$ and $\mathcal{U} m=\mathcal{U} n=q, \mathcal{U} p=\mathcal{U} q=p$. We have, in this case, 


\begin{tabular}{lcccc}
\hline $\mathrm{v}$ & $m$ & $n$ & $p$ & $q$ \\
\hline$Z \mathrm{v}$ & $\mathrm{p}$ & $\mathrm{q}$ & $\mathrm{p}$ & $\mathrm{p}$ \\
$\mathcal{U} \mathrm{v}$ & $\mathrm{q}$ & $\mathrm{q}$ & $\mathrm{p}$ & $\mathrm{p}$ \\
$d_{\mathrm{b}}(\mathrm{v}, Z \mathrm{v})$ & 5 & 5 & 0 & 1 \\
$d_{\mathrm{b}}(\mathrm{v}, \mathcal{U} \mathrm{v})$ & 7 & 5 & 0 & 1 \\
\hline
\end{tabular}

\begin{tabular}{|c|c|c|c|c|c|c|}
\hline \multirow{2}{*}{\multicolumn{2}{|c|}{$d_{\mathrm{b}}(Z \mathrm{v}, \mathcal{U} \mathrm{w})$}} & \multirow{3}{*}{$\frac{\mathrm{v}}{z \mathrm{v}}$} & \multirow{3}{*}{$\frac{m}{\mathrm{p}}$} & \multirow{3}{*}{$\frac{n}{q}$} & \multirow{3}{*}{$\frac{p}{p}$} & \multirow{3}{*}{$\frac{q}{p}$} \\
\hline & & & & & & \\
\hline W & $\mathcal{U w}$ & & & & & \\
\hline $\mathrm{m}$ & $\mathrm{q}$ & & 1 & 0 & 1 & 1 \\
\hline $\mathrm{n}$ & $\mathrm{q}$ & & 1 & 0 & 1 & 1 \\
\hline $\mathrm{p}$ & $\mathrm{p}$ & & 0 & 1 & 0 & 0 \\
\hline q & $\mathrm{p}$ & & 0 & 1 & 0 & 0 \\
\hline
\end{tabular}

Letting the functions $\Psi, \Phi \in \Theta, \Psi(\theta)=\theta, \Phi(\theta)=\frac{3}{4} \theta$ and the numbers $\alpha=2, c_{1}=1$, $c_{2}=1, c_{3}=c_{4}=c_{5}=\frac{1}{4}$, we can easily see that assumptions $\left(\beta_{1}\right)$ and $\left(\beta_{2}\right)$ in Theorem 13 are satisfied. We show that (13) is satisfied for any pair $(v, w) \in \mathcal{L}$, where

$$
\mathcal{L}=\{(m, n),(n, p),(n, q),(p, m),(p, n),(q, m),(q, n)\}
$$

(the other cases are excluded by the hypotheses of Theorem 13).

- $(\mathrm{v}, \mathrm{w})=(m, n)$

$$
\begin{aligned}
s^{\alpha} d_{\mathrm{b}}(Z \mathrm{v}, \mathcal{U l} \mathrm{w}) \\
=2^{2} d_{\mathrm{b}}(Z m, \mathcal{U} n) \\
=4 \leq \frac{154}{16}=\frac{3}{4}\left(c_{1} d_{\mathrm{b}}(m, n)+c_{2} d_{\mathrm{b}}(m, Z m)\right. \\
\left.\quad+c_{3} d_{\mathrm{b}}(n, \mathcal{U} n)+c_{4} \frac{d_{\mathrm{b}}(n, \mathcal{U} \mathrm{l} n) d_{\mathrm{b}}(m, Z m)}{1+d_{\mathrm{b}}(m, n)}+c_{5} \frac{d_{\mathrm{b}}(n, \mathcal{U} n)\left(1+d_{\mathrm{b}}(m, Z m)\right)}{1+d_{\mathrm{b}}(m, n)}\right)
\end{aligned}
$$

- $(\mathrm{v}, \mathrm{w})=(n, p)$

$$
\begin{aligned}
2^{2} d_{\mathrm{b}}(Z n, \mathcal{U} p) \\
=4 \leq \frac{39}{4}=\frac{3}{4}(8+5) \\
=\frac{3}{4}\left(c_{1} d_{\mathrm{b}}(n, p)+c_{2} d_{\mathrm{b}}(n, Z n)\right. \\
\left.\quad+c_{3} d_{\mathrm{b}}(p, \mathcal{U} p)+c_{4} \frac{d_{\mathrm{b}}(p, \mathcal{U} p) d_{\mathrm{b}}(n, Z n)}{1+d_{\mathrm{b}}(n, p)}+c_{5} \frac{d_{\mathrm{b}}(p, \mathcal{U} p)\left(1+d_{\mathrm{b}}(n, Z n)\right)}{1+d_{\mathrm{b}}(n, p)}\right) .
\end{aligned}
$$

The other cases are discussed similarly.

Thus $\mathcal{F}_{\mathrm{S}}(Z, \mathcal{U})=\{p\}$.

Theorem 16 Let $\left(\mathrm{S}, d_{\mathrm{b}}, \mathrm{s}\right)$ be a complete $d_{\mathrm{b}}-\mathrm{ms}$, the functions $\Psi, \Phi \in \Theta$, a number $\alpha \in$ $[1, \infty), c_{1}>0, c_{2} \geq 0$, and two mappings $z, \mathfrak{u}: \mathrm{S} \rightarrow \mathrm{S}$ such that, for every distinct $\mathrm{v}, \mathrm{w} \in \mathrm{S}$ 
with $d_{\mathrm{b}}(Z \mathrm{v}, \mathcal{U} \mathrm{w})>0$, the following inequality

$$
\Psi\left(\mathrm{s}^{\alpha} d_{\mathrm{b}}(Z \mathrm{v}, \mathcal{U} \mathrm{w})\right) \leq \Phi\left(R_{3}(\mathrm{v}, \mathrm{w})\right)
$$

holds, where

$$
R_{3}(\mathrm{v}, \mathrm{w})=c_{1} d_{\mathrm{b}}(\mathrm{v}, \mathrm{w})+c_{2} \frac{d_{\mathrm{b}}(\mathrm{v}, Z \mathrm{v}) d_{\mathrm{b}}(\mathrm{v}, \mathcal{U} \mathrm{w})+d_{\mathrm{b}}(\mathrm{w}, \mathcal{U} \mathrm{w}) d_{\mathrm{b}}(\mathrm{w}, Z \mathrm{v})}{1+4 \max \left\{d_{\mathrm{b}}(\mathrm{v}, Z \mathrm{v}), d_{\mathrm{b}}(\mathrm{w}, \mathcal{U} \mathrm{w})\right\}}
$$

\section{Assume that:}

$\left(\beta_{1}\right) \quad c_{1}+\mathrm{s} c_{2}<\mathrm{s}^{\alpha}$

$\left(\beta_{2}\right) \Psi$ is nondecreasing.

Then the set $\mathcal{F}_{\mathrm{S}}(\mathcal{Z}, \mathcal{U})$ has exactly one element.

Proof Let us take in (20), $\mathrm{v}=\mathrm{v}_{2 n}$ and $\mathrm{w}=\mathrm{v}_{2 n+1}$, where the sequence $\left\{\mathrm{v}_{n}\right\}$ is defined as in Theorem 11. We have

$$
\Psi\left(\mathrm{s}^{\alpha} d_{\mathrm{b}}\left(Z \mathrm{v}_{2 n}, \mathcal{U} \mathrm{v}_{2 n+1}\right)\right) \leq \Phi\left(R_{3}\left(\mathrm{v}_{2 n}, \mathrm{v}_{2 n+1}\right)\right)<\Psi\left(R_{3}\left(\mathrm{v}_{2 n}, \mathrm{v}_{2 n+1}\right)\right)
$$

with

$$
\begin{aligned}
& R_{3}\left(\mathrm{v}_{2 n}, \mathrm{v}_{2 n+1}\right) \\
&=c_{1} d_{\mathrm{b}}\left(\mathrm{v}_{2 n}, \mathrm{v}_{2 n+1}\right)+c_{2} \frac{d_{\mathrm{b}}\left(\mathrm{v}_{2 n}, Z \mathrm{v}_{2 n}\right) d_{\mathrm{b}}\left(\mathrm{v}_{2 n}, \mathcal{U} \mathrm{v}_{2 n+1}\right)+d_{\mathrm{b}}\left(\mathrm{v}_{2 n+1}, \mathcal{u} \mathrm{v}_{2 n+1}\right) d_{\mathrm{b}}\left(\mathrm{v}_{2 n+1}, Z \mathrm{v}_{2 n}\right)}{1+4 \max \left\{d_{\mathrm{b}}\left(\mathrm{v}_{2 n}, Z \mathrm{v}_{2 n}\right), d_{\mathrm{b}}\left(\mathrm{v}_{2 n+1}, \mathcal{U} \mathrm{v}_{2 n+1}\right)\right\}} \\
&=c_{1} d_{\mathrm{b}}\left(\mathrm{v}_{2 n}, \mathrm{v}_{2 n+1}\right)+c_{2} \frac{d_{\mathrm{b}}\left(\mathrm{v}_{2 n}, \mathrm{v}_{2 n+1}\right) d_{\mathrm{b}}\left(\mathrm{v}_{2 n}, \mathrm{v}_{2 n+2}\right)+d_{\mathrm{b}}\left(\mathrm{v}_{2 n+1}, \mathrm{v}_{2 n+2}\right) d_{\mathrm{b}}\left(\mathrm{v}_{2 n+1}, \mathrm{v}_{2 n+1}\right)}{1+4 \max \left\{d_{\mathrm{b}}\left(\mathrm{v}_{2 n}, \mathrm{v}_{2 n+1}\right), d_{\mathrm{b}}\left(\mathrm{v}_{2 n+1}, \mathrm{v}_{2 n+2}\right)\right\}} \\
&=c_{1} d_{\mathrm{b}}\left(\mathrm{v}_{2 n}, \mathrm{v}_{2 n+1}\right) \\
&+c_{2} \frac{\mathrm{s} d_{\mathrm{b}}\left(\mathrm{v}_{2 n}, \mathrm{v}_{2 n+1}\right)\left(d_{\mathrm{b}}\left(\mathrm{v}_{2 n}, \mathrm{v}_{2 n+1}\right)+d_{\mathrm{b}}\left(\mathrm{v}_{2 n+1}, \mathrm{v}_{2 n+2}\right)\right)+2 \mathrm{~s} d_{\mathrm{b}}\left(\mathrm{v}_{2 n+1}, \mathrm{v}_{2 n+2}\right) d_{\mathrm{b}}\left(\mathrm{v}_{2 n}, \mathrm{v}_{2 n+1}\right)}{1+4 \max \left\{d_{\mathrm{b}}\left(\mathrm{v}_{2 n}, \mathrm{v}_{2 n+1}\right), d_{\mathrm{b}}\left(\mathrm{v}_{2 n+1}, \mathrm{v}_{2 n+2}\right)\right\}} \\
&=c_{1} d_{\mathrm{b}}\left(\mathrm{v}_{2 n}, \mathrm{v}_{2 n+1}\right) \\
&+\mathrm{s} c_{2} d_{\mathrm{b}}\left(\mathrm{v}_{2 n}, \mathrm{v}_{2 n+1}\right) \frac{d_{\mathrm{b}}\left(\mathrm{v}_{2 n}, \mathrm{v}_{2 n+1}\right)+3 d_{\mathrm{b}}\left(\mathrm{v}_{2 n+1}, \mathrm{v}_{2 n+2}\right)}{1+4 \max \left\{d_{\mathrm{b}}\left(\mathrm{v}_{2 n}, \mathrm{v}_{2 n+1}\right), d_{\mathrm{b}}\left(\mathrm{v}_{2 n+1}, \mathrm{v}_{2 n+2}\right)\right\}} \\
& \leq\left(c_{1}+\mathrm{s} c_{2}\right) d_{\mathrm{b}}\left(\mathrm{v}_{2 n}, \mathrm{v}_{2 n+1}\right) .
\end{aligned}
$$

Furthermore, taking $\left(\beta_{2}\right)$ and the above relation into account, we get

$$
\mathrm{s}^{\alpha} d_{\mathrm{b}}\left(\mathrm{v}_{2 n+1}, \mathrm{v}_{2 n+2}\right)=\mathrm{s}^{\alpha} d_{\mathrm{b}}\left(Z \mathrm{v}_{2 n}, \mathcal{U} \mathrm{v}_{2 n+1}\right)<R_{3}\left(\mathrm{v}_{2 n}, \mathrm{v}_{2 n+1}\right) \leq\left(c_{1}+\mathrm{sc}_{2}\right) d_{\mathrm{b}}\left(\mathrm{v}_{2 n}, \mathrm{v}_{2 n+1}\right),
$$

which implies

$$
d_{b}\left(\mathrm{v}_{2 n+1}, \mathrm{v}_{2 n+2}\right)<\frac{\mathcal{c}_{1}+\mathrm{S} \mathcal{c}_{2}}{\mathrm{~S}^{\alpha}} d_{\mathrm{b}}\left(\mathrm{v}_{2 n}, \mathrm{v}_{2 n+1}\right)
$$

Similarly, taking $\mathrm{v}=\mathrm{v}_{2 n}$, respectively $\mathrm{w}=\mathrm{v}_{2 n-1}$, we obtain

$$
d_{\mathrm{b}}\left(\mathrm{v}_{2 n+1}, \mathrm{v}_{2 n}\right)<\frac{\mathcal{c}_{1}+\mathrm{S} c_{2}}{\mathrm{~S}^{\alpha}} d_{\mathrm{b}}\left(\mathrm{v}_{2 n}, \mathrm{v}_{2 n-1}\right) .
$$


Now, choosing $\mathcal{C}=\frac{c_{1}+s_{2}}{s^{\alpha}}<1$ (by assumption $\left.\left(\beta_{1}\right)\right)$, we have $d_{\mathrm{b}}\left(\mathrm{v}_{n}, \mathrm{v}_{n+1}\right)<\mathcal{C} d_{\mathrm{b}}\left(\mathrm{v}_{n-1}, \mathrm{v}_{n}\right)$ for any $n \in \mathbb{N}$. Therefore, Lemma 10 leads us to the conclusion that $\left\{\mathrm{v}_{n}\right\}$ is a Cauchy sequence. Thus, since the space is complete, there exists $x \in S$ such that

$$
\lim _{n, p \rightarrow \infty} d_{\mathrm{b}}\left(\mathrm{v}_{n}, \mathrm{v}_{p}\right)=0=\lim _{n \rightarrow \infty} d_{\mathrm{b}}\left(\mathrm{v}_{n}, \mathrm{x}\right)
$$

Supposing that $z x \neq x$, we have

$$
d_{\mathrm{b}}(Z \mathrm{x}, \mathrm{x}) \leq \mathrm{s}\left[d_{\mathrm{b}}\left(Z \mathrm{x}, \mathrm{v}_{2 n}\right)+d_{\mathrm{b}}\left(\mathrm{v}_{2 n}, \mathrm{x}\right)\right]=\mathrm{s}\left[d_{\mathrm{b}}\left(Z \mathrm{x}, \mathcal{U} \mathrm{v}_{2 n-1}\right)+d_{\mathrm{b}}\left(\mathrm{v}_{2 n}, \mathrm{x}\right)\right]
$$

Moreover, without loss of generality, we can assume that $d_{\mathrm{b}}\left(\mathrm{v}_{n}, \mathrm{x}\right)>0$ for any $n \in \mathbb{N}$, and then from (20) we get

$$
\Psi\left(\mathrm{s}^{\alpha}\left(Z \mathrm{x}, \mathcal{U} \mathrm{v} \mathrm{v}_{2 n-1}\right)\right) \leq \Phi\left(R_{3}\left(\mathrm{x}, \mathrm{v}_{2 n-1}\right)\right)<\Psi\left(R_{3}\left(\mathrm{x}, \mathrm{v}_{2 n-1}\right)\right)
$$

or, by $\left(\beta_{2}\right)$,

$$
\begin{aligned}
& d_{\mathrm{b}}\left(Z \mathrm{x}, \mathcal{U} \mathrm{v}_{2 n-1}\right) \\
& \left.\quad \leq \mathrm{s}^{\alpha} d_{\mathrm{b}}\left(Z \mathrm{x}, \mathcal{U} \mathrm{v}_{2 n-1}\right)<R_{3}\left(\mathrm{x}, \mathrm{v}_{2 n-1}\right)\right) \\
& \quad=c_{1} d_{\mathrm{b}}\left(\mathrm{x}, \mathrm{v}_{2 n-1}\right)++c_{2} \frac{d_{\mathrm{b}}(\mathrm{x}, Z \mathrm{x}) d_{\mathrm{b}}\left(\mathrm{x}, \mathcal{U} \mathrm{v}_{2 n-1}\right)+d_{\mathrm{b}}\left(\mathrm{v}_{2 n-1}, \mathcal{U} \mathrm{v}_{2 n-1}\right) d_{\mathrm{b}}\left(\mathrm{v}_{2 n-1}, Z \mathrm{x}\right)}{1+4 \max \left\{d_{\mathrm{b}}(\mathrm{x}, Z \mathrm{x}), d_{\mathrm{b}}\left(\mathrm{v}_{2 n-1}, \mathcal{U} \mathrm{v}_{2 n-1}\right)\right\}} \\
& \quad=c_{1} d_{\mathrm{b}}\left(\mathrm{x}, \mathrm{v}_{2 n-1}\right)+c_{2} \frac{d_{\mathrm{b}}(\mathrm{x}, Z \mathrm{x}) d_{\mathrm{b}}\left(\mathrm{x}, \mathrm{v}_{2 n}\right)+d_{\mathrm{b}}\left(\mathrm{v}_{2 n-1}, \mathrm{v}_{2 n}\right) d_{\mathrm{b}}\left(\mathrm{v}_{2 n-1}, Z \mathrm{x}\right)}{1+4 \max \left\{d_{\mathrm{b}}(\mathrm{x}, Z \mathrm{x}), d_{\mathrm{b}}\left(\mathrm{v}_{2 n-1}, \mathrm{v}_{2 n}\right)\right\}}
\end{aligned}
$$

Returning in (25), we have

$$
\begin{aligned}
d_{\mathrm{b}}(Z \mathrm{x}, \mathrm{x})<\mathrm{s}\left[c_{1} d_{\mathrm{b}}\left(\mathrm{x}, \mathrm{v}_{2 n-1}\right)+c_{2} \frac{d_{\mathrm{b}}(\mathrm{x}, Z \mathrm{x}) d_{\mathrm{b}}\left(\mathrm{x}, \mathrm{v}_{2 n}\right)+d_{\mathrm{b}}\left(\mathrm{v}_{2 n-1}, \mathrm{v}_{2 n}\right) d_{\mathrm{b}}\left(\mathrm{v}_{2 n-1}, Z \mathrm{x}\right)}{1+4 \max \left\{d_{\mathrm{b}}(\mathrm{x}, Z \mathrm{x}), d_{\mathrm{b}}\left(\mathrm{v}_{2 n-1}, \mathrm{v}_{2 n}\right)\right\}}\right. \\
\left.+d_{\mathrm{b}}\left(\mathrm{v}_{2 n}, \mathrm{x}\right)\right] .
\end{aligned}
$$

Letting $n \rightarrow \infty$ and keeping in mind (24), we get

$$
d_{\mathrm{b}}(Z \mathrm{x}, \mathrm{x})<0
$$

which is a contradiction. Thus, $d_{\mathrm{b}}(Z \mathrm{x}, \mathrm{x})=0$ and from $\left(d_{\mathrm{b}_{1}}\right)$ we have $\mathrm{x}=Z \mathrm{x}$.

Analogously, we have

$$
\Psi\left(\mathrm{s}^{\alpha} d_{\mathrm{b}}\left(z \mathrm{v}_{2 n}, \mathcal{U} \mathrm{x}\right)\right) \leq \Phi\left(R_{3}\left(\mathrm{v}_{2 n}, \mathrm{x}\right)\right)<\Psi\left(R_{3}\left(\mathrm{v}_{2 n}, \mathrm{x}\right)\right)
$$

or, by $\left(\beta_{2}\right)$,

$$
\begin{aligned}
d_{\mathrm{b}}\left(Z \mathrm{v}_{2 n}, \mathcal{U} \mathrm{x}\right) & <R_{3}\left(\mathrm{v}_{2 n}, \mathrm{x}\right) \\
& =c_{1} d_{\mathrm{b}}\left(\mathrm{x}, \mathrm{v}_{2 n}\right)+c_{2} \frac{d_{\mathrm{b}}\left(\mathrm{v}_{2 n}, \mathrm{v}_{2 n+1}\right) d_{\mathrm{b}}\left(\mathrm{v}_{2 n}, \mathcal{U} \mathrm{x}\right)+d_{\mathrm{b}}(\mathrm{x}, \mathcal{U} \mathrm{x}) d_{\mathrm{b}}\left(\mathrm{x}, \mathrm{v}_{2 n+1}\right)}{1+4 \max \left\{d_{\mathrm{b}}(\mathrm{x}, \mathcal{U} \mathrm{x}), d_{\mathrm{b}}\left(\mathrm{v}_{2 n}, \mathrm{v}_{2 n+1}\right)\right\}}
\end{aligned}
$$


On the other hand, supposing that $d_{b}(x, \mathcal{U} x)>0$, we have

$$
0<d_{\mathrm{b}}(\mathrm{x}, \mathcal{U} \mathrm{x}) \leq \mathrm{s}\left[d_{\mathrm{b}}\left(\mathrm{x}, \mathrm{v}_{2 n+1}\right)+d_{\mathrm{b}}\left(\mathrm{v}_{2 n+1}, \mathcal{U} \mathrm{x}\right)\right]=\mathrm{s}\left[d_{\mathrm{b}}\left(\mathrm{x}, \mathrm{v}_{2 n+1}\right)+d_{\mathrm{b}}\left(Z \mathrm{v}_{2 n}, \mathcal{U} \mathrm{x}\right)\right]
$$

Combining the above inequalities and taking limit as $n \rightarrow \infty$, we obtain $0<d_{b}(x, \mathcal{U} x)<0$, which is a contradiction. Therefore, $d_{b}(x, \mathcal{u x})=0$, and then $x=\mathcal{U} x$. Thus, $\mathrm{x}$ is a common fixed point for $Z$ and $\mathcal{U}$, that is, $\mathcal{F}_{\mathrm{S}}(Z, \mathcal{U}) \neq \emptyset$ and it remains to show that the set $\mathcal{F}_{\mathrm{S}}(Z, \mathcal{U})$ is in fact reduced to a single point. On the contrary, let $v \in \mathcal{F}_{\mathrm{S}}(Z, \mathcal{U})$ with $v \neq \mathrm{x}$. Replaced in (20), we have

$$
\Psi\left(\mathrm{s}^{\alpha} d_{\mathrm{b}}(Z \mathrm{x}, \mathcal{U} v)\right) \leq \Phi\left(R_{3}(\mathrm{x}, v)\right)<\Psi\left(R_{3}(\mathrm{x}, v)\right)
$$

and, due to $\left(\beta_{2}\right)$,

$$
\begin{aligned}
\mathrm{s}^{\alpha} d_{\mathrm{b}}(\mathrm{x}, v) & \left.=\mathrm{s}^{\alpha} d_{\mathrm{b}}(Z \mathrm{x}, \mathcal{U} v)\right)<\left(R_{3}(\mathrm{x}, v)\right. \\
& =c_{1} d_{\mathrm{b}}(\mathrm{x}, v)+c_{2} d_{\mathrm{b}} \frac{d_{\mathrm{b}}(\mathrm{x}, Z \mathrm{x}) d_{\mathrm{b}}(\mathrm{x}, \mathcal{U} v)+d_{\mathrm{b}}(v, \mathcal{U} v) d_{\mathrm{b}}(v, Z \mathrm{x})}{1+4 \max \left\{d_{\mathrm{b}}(\mathrm{x}, Z \mathrm{x}), d_{\mathrm{b}}(v, \mathcal{U} v)\right\}} \\
& =c_{1} d_{\mathrm{b}}(\mathrm{x}, v),
\end{aligned}
$$

which is a contradiction. Therefore, it follows that $\mathrm{x}=v$ and the set $\mathcal{F}_{\mathrm{S}}(Z, \mathcal{U})$ has exactly one element.

Example 17 Let $S=\{2,4,5,7\}$ and two self-mappings $\mathcal{U}, Z$ be defined on $S$ by

\begin{tabular}{lllll}
\hline $\mathrm{v}$ & 2 & 4 & 5 & 7 \\
\hline$Z \mathrm{v}$ & 5 & 5 & 5 & 4 \\
$\mathcal{U} \mathrm{w}$ & 4 & 5 & 5 & 5
\end{tabular}

Let $d_{\mathrm{b}}$ be the $d_{\mathrm{b}}$-metric on $S$ (with $\mathrm{s}=2$ ) given by

$$
d_{\mathrm{b}}(v, w)= \begin{cases}3 & \text { if } v=w=4 \\ |v-w|^{2} & \text { otherwise }\end{cases}
$$

Considering the functions $\Psi, \Phi \in \Theta$ as in Example (15) and letting $\alpha=2, c_{1}=2, c_{2}=\frac{3}{4}$, we have the following cases:

- $(\mathrm{v}, \mathrm{w})=(4,2)$

$$
\begin{aligned}
4 d_{\mathrm{b}}(z 4, \mathcal{U} 2) & =4 d_{\mathrm{b}}(5,4)=4<6 \leq \frac{3}{4}\left(8+\frac{3}{4} \cdot \frac{39}{17}\right) \\
& =\frac{3}{4}\left[2 \cdot 4+\frac{3}{4} \cdot \frac{1 \cdot 3+4 \cdot 9}{1+4 \cdot 4}\right]=\frac{3}{4} R_{3}(4,2)
\end{aligned}
$$

- $(\mathrm{v}, \mathrm{w})=(5,2)$

$$
4 d_{\mathrm{b}}(z 5, \mathcal{U} 2)=4 d_{\mathrm{b}}(5,4)=4<\frac{27}{2}=\frac{3}{4} \cdot 2 d_{\mathrm{b}}(5,2) \leq \frac{3}{4} R_{3}(5,2)
$$


- $(\mathrm{v}, \mathrm{w})=(7,2)$

$$
4 d_{\mathrm{b}}(z 7, \mathcal{Z} 2)=4 d_{\mathrm{b}}(4,4)=4 \cdot 3=12<\frac{75}{2}=\frac{3}{4} \cdot 2 d_{\mathrm{b}}(7,2) \leq \frac{3}{4} R_{3}(7,2) ;
$$

- $(v, w)=(7,4)$

$$
4 d_{\mathrm{b}}(z 7, \mathfrak{u} 4)=4 d_{\mathrm{b}}(4,5)=4<6=\frac{3}{4} \cdot 2 d_{\mathrm{b}}(7,4) \leq \frac{3}{4} R_{3}(7,4)
$$

- $(\mathrm{v}, \mathrm{w})=(7,5)$

$$
4 d_{\mathrm{b}}(z 7, \mathcal{u})=4 d_{\mathrm{b}}(4,5)=4<\frac{27}{2}=\frac{3}{4} \cdot 2 d_{\mathrm{b}}(7,5) \leq \frac{3}{4} R_{3}(7,4) .
$$

The other cases are excluded by the hypothesis of Theorem 16 . Therefore, $\mathcal{F}_{\mathrm{S}}(Z, \mathcal{U})=\{5\}$.

Corollary 18 Let $\left(\mathrm{S}, d_{\mathrm{b}}, \mathrm{s}\right)$ be a complete $d_{\mathrm{b}}-\mathrm{ms}, \Psi, \Phi \in \Theta$, a number $\alpha \in[1, \infty), c_{1}>0, c_{2} \geq$ 0 , and a mapping $Z: S \rightarrow S$ such that, for every distinct $\mathrm{v}, \mathrm{w} \in \mathrm{S}$ with $\alpha_{\mathrm{b}}(Z \mathrm{v}, Z \mathrm{w})>0$, the following inequality

$$
\Psi\left(\mathrm{s}^{\alpha} d_{\mathrm{b}}(\mathrm{Zv}, \mathrm{zw})\right) \leq \Phi\left(R_{3}(\mathrm{v}, \mathrm{w})\right)
$$

holds, where

$$
R_{3}(\mathrm{v}, \mathrm{w})=c_{1} d_{\mathrm{b}}(\mathrm{v}, \mathrm{w})+c_{2} \frac{d_{\mathrm{b}}(\mathrm{v}, Z \mathrm{v}) d_{\mathrm{b}}(\mathrm{v}, Z \mathrm{w})+d_{\mathrm{b}}(\mathrm{w}, Z \mathrm{w}) d_{\mathrm{b}}(\mathrm{w}, Z \mathrm{v})}{1+4 \max \left\{d_{\mathrm{b}}(\mathrm{v}, Z \mathrm{v}), d_{\mathrm{b}}(\mathrm{w}, Z \mathrm{w})\right\}}
$$

Assume that:

$\left(\beta_{1}\right) c_{1}+\mathrm{s} c_{2}<\mathrm{s}^{\alpha}$

$\left(\beta_{2}\right) \Psi$ is nondecreasing.

Then the set $\mathcal{F}_{\mathrm{S}}(Z)$ has exactly one element.

Proof Let $z=\mathcal{U}$ in Theorem 16 .

\section{Consequences}

Taking particular functions $\Psi$ and $\Phi$, we obtain as consequences some known results. For example, let $\Phi(\theta)=\beta(\theta) \Psi(\theta)$ for all $\theta>0$ and $\beta:[0, \infty) \rightarrow\left[0, \frac{1}{5}\right)$.

Corollary 19 Let $\left(\mathrm{S}, d_{\mathrm{b}}, \mathrm{s}\right)$ be a complete $d_{\mathrm{b}}-\mathrm{ms}$, a number $\alpha \in[1, \infty)$, and two continuous mappings $Z, \mathcal{U}: \mathrm{S} \rightarrow \mathrm{S}$ such that, for every distinct $\mathrm{v}, \mathrm{w} \in \mathrm{S}$ with $d_{\mathrm{b}}(Z \mathrm{v}, \mathcal{U} \mathrm{w})>0$, the following inequality

$$
\Psi\left(\mathrm{s}^{\alpha} d_{\mathrm{b}}(Z \mathrm{v}, \mathcal{U} \mathrm{w})\right) \leq \beta\left(R_{1}(\mathrm{v}, \mathrm{w})\right) \Psi\left(R_{1}(\mathrm{v}, \mathrm{w})\right)
$$

holds. Assume that:

$\left(\beta_{1}\right) c_{1}+c_{2}+c_{3}+c_{4}<s^{\alpha}$ and $c_{1}>0$

$\left(\beta_{3}\right) \Psi:(0, \infty) \rightarrow(0, \infty)$ is nondecreasing;

$\left(\beta_{4}\right) \beta:(0, \infty) \rightarrow\left(0, \frac{1}{s}\right)$ satisfies $\lim \sup _{\theta \rightarrow \theta_{0}} \beta(\theta)<\frac{1}{\mathrm{~s}}$ for any $\theta_{0}>0$. 
Then $\mathcal{F}_{\mathrm{S}}(\mathcal{Z}, \mathcal{U}) \neq \emptyset$. Moreover, if $c_{1}+2 c_{2}+2 c_{3}+4 c_{4} \leq \mathrm{s}^{\alpha}$, then the set $\mathcal{F}_{\mathrm{S}}(\mathcal{Z}, \mathcal{U})$ has exactly one element.

Corollary 20 Let $\left(\mathrm{S}, d_{\mathrm{b}}, \mathrm{s}\right)$ be a complete $d_{\mathrm{b}}-\mathrm{ms}$, a number $\alpha \in[1, \infty)$, and two mappings $Z, \mathcal{U}: \mathrm{S} \rightarrow \mathrm{S}$ such that, for every distinct $\mathrm{v}, \mathrm{w} \in \mathrm{S}$ with $\alpha_{\mathrm{b}}(z \mathrm{v}, \mathcal{u} \mathrm{w})>0$, the following inequality

$$
\Psi\left(\mathrm{s}^{\alpha} d_{\mathrm{b}}(Z \mathrm{v}, \tau \mathrm{w})\right) \leq \Phi\left(R_{2}(\mathrm{v}, \mathrm{w})\right)
$$

holds. Assume that:

$\left(\beta_{1}\right) c_{1}+c_{2}+c_{3}+c_{4}+c_{5}<\mathrm{s}^{\alpha}, c_{1}>0, c_{2} \leq 1$, and $c_{3}+c_{5} \leq 1$;

$\left(\beta_{3}\right) \Psi:(0, \infty) \rightarrow(0, \infty)$ is nondecreasing;

$\left(\beta_{4}\right) \beta:(0, \infty) \rightarrow\left(0, \frac{1}{s}\right)$ satisfies $\lim \sup _{\theta \rightarrow \theta_{0}} \beta(\theta)<\frac{1}{\mathrm{~s}}$ for any $\theta_{0}>0$.

Then $\mathcal{F}_{S}(\mathcal{Z}, \mathcal{U}) \neq \emptyset$. Moreover, if $c_{1} \leq 1$, then the set $\mathcal{F}_{S}(Z, \mathcal{U})$ has exactly one element.

Corollary 21 Let $\left(\mathrm{S}, d_{\mathrm{b}}, \mathrm{s}\right)$ be a complete $d_{\mathrm{b}}-\mathrm{ms}$, a number $\alpha \in[1, \infty)$, and two mappings $z, \mathcal{u}: \mathrm{S} \rightarrow \mathrm{S}$ such that, for every distinct $\mathrm{v}, \mathrm{w} \in \mathrm{S}$ with $\alpha_{\mathrm{b}}(z \mathrm{v}, \mathfrak{u} \mathrm{w})>0$, the following inequality

$$
\Psi\left(\mathrm{s}^{\alpha} d_{\mathrm{b}}(Z \mathrm{v}, \mathcal{U} \mathrm{w})\right) \leq \Phi\left(R_{3}(\mathrm{v}, \mathrm{w})\right)
$$

\section{holds. Assume that:}

$\left(\beta_{1}\right) c_{1}+\mathrm{sc}_{2}<\mathrm{s}^{\alpha}, c_{1}>0$

$\left(\beta_{3}\right) \Psi:(0, \infty) \rightarrow(0, \infty)$ is nondecreasing;

$\left(\beta_{4}\right) \beta:(0, \infty) \rightarrow\left(0, \frac{1}{s}\right)$ satisfies $\lim \sup _{\theta \rightarrow \theta_{0}} \beta(\theta)<\frac{1}{\mathrm{~s}}$ for any $\theta_{0}>0$.

Then the set $\mathcal{F}_{\mathrm{S}}(\mathcal{Z}, \mathcal{U})$ has exactly one element.

Considering $\Phi(\theta)=\kappa \Psi(\theta)$ or $\Phi(\theta)=\kappa \cdot \theta$ for all $\theta>0$ in Theorems 11, 13 or 16, other consequences can be listed. On the other hand, many other corollaries can be deduced considering $z=\mathcal{U}$ or letting $\mathrm{s}=1$.

\section{Acknowledgements}

The authors thank their universities. The authors extend their appreciation to the Deanship of Scientific Research at King Saud University for funding this work through research group no. RG-1437-037. A.F. Roldán López de Hierro is grateful to Project TIN2017-89517-P of Ministerio de Economía, Industria y Competitividad and also to Junta de Andalucía by project FQM-365 of the Andalusian CICYE.

\section{Funding}

This research received no external funding.

Availability of data and materials

The data and material used to support the findings of this study are included within the article.

\section{Competing interests}

The authors declare that they have no competing interests.

\section{Authors' contributions}

The authors carried out the whole manuscript. All authors read and approved the final manuscript.

\section{Author details}

'Department of Mathematics, King Saud University, Riyadh, Saudi Arabia. ${ }^{2}$ Department of Mathematics and Computer Sciences, Transilvania University of Brasov, Brasov, Romania. ${ }^{3}$ Department of Statistics and Operations Research, University of Granada, Facultad de Farmacia, Campus de Cartuja, s/n, 18071, Granada, Spain. 


\section{Publisher's Note}

Springer Nature remains neutral with regard to jurisdictional claims in published maps and institutional affiliations.

\section{Received: 18 January 2021 Accepted: 2 March 2021 Published online: 10 March 2021}

\section{References}

1. Afshari, H., Aydi, H., Karapınar, E.: On generalized $\alpha$ - $\psi$-Geraghty contractions on b-metric spaces. Georgian Math. J. 27, 9-21 (2020)

2. Alghamdi, M.A., Gulyaz-Ozyurt, S., Karapınar, E.: A note on extended Z-contraction. Mathematics 2020(8), 195 (2020)

3. Alsulami, H., Gulyaz, S., Karapınar, E., Erhan, I.: An Ulam stability result on quasi-b-metric-like spaces. Open Math. (2016). https://doi.org/10.1515/math-2016-0097

4. Aksoy, Ü., Karapınar, E., Erhan, I.M.: Fixed points of generalized $\alpha$-admissible contractions on $b$-metric spaces with an application to boundary value problems. J. Nonlinear Convex Anal. 17, 1095-1108 (2016)

5. Aydi, H., Karapınar, E., Bota, M.F., Mitrović, S.: A fixed point theorem for set-valued quasi-contractions in b-metric spaces. Fixed Point Theory Appl. 2012, 88 (2012)

6. Aydi, H., Bota, M.F., Karapınar, E., Moradi, S.: A common fixed point for weak $\phi$-contractions on $b$-metric spaces. Fixed Point Theory 13, 337-346 (2012)

7. Fulga, A., Karapınar, E., Petruşel, G.: On hybrid contractions in the context of quasi-metric spaces. Mathematics 2020(8), $675(2020)$

8. Gulyaz-Ozyurt, S.: On some $\alpha$-admissible contraction mappings on Branciari $b$-metric spaces. Adv. Theor. Nonlinear Anal. Appl. 1, 1-13 (2017)

9. Karapınar, E., Chifu, C.: Results in wt-distance over b-metric spaces. Mathematics 2020(8), 220 (2020)

10. Karapınar, E., Fulga, A., Petruşel, A.: On Istratescu type contractions in $b$-metric spaces. Mathematics 2020(8), 388 (2020)

11. Marasi, H.R., Aydi, H.: Existence and uniqueness results for two-term nonlinear fractional differential equations via a fixed point technique. J. Math. 2021, Article ID 6670176 (2021)

12. Javed, K., Aydi, H., Uddin, F., Arshad, M.: On orthogonal partial b-metric spaces with an application. J. Math. 2021, Article ID 6692063 (2021)

13. Mlaiki, N., Souayah, N., Abdeljawad, T., Aydi, H.: A new extension to the controlled metric type spaces endowed with a graph. Adv. Differ. Equ. 2021(2021), 94 (2021)

14. Hammad, H.A., Aydi, H., Mlaiki, N.: Contributions of the fixed point technique to solve the 2D Volterra integral equations, Riemann-Liouville fractional integrals, and Atangana-Baleanu integral operators. Adv. Differ. Equ. 2021(2021), 97 (2021)

15. Aydi, H., Lakzian, H., Mitrović, Z.D., Radenović, S.: Best proximity points of MT-cyclic contractions with property UC. Numer. Funct. Anal. Optim. 41(7), 871-882 (2020)

16. Karapınar, E., Czerwik, S., Aydi, H.: $(\alpha, \psi)$-Meir-Keeler contraction mappings in generalized $b$-metric spaces. J. Funct. Spaces 2018, Article ID 3264620 (2018)

17. Alqahtani, B., Fulga, A., Karapınar, E., Kumari, P.S.: Sehgal type contractions on dislocated spaces. Mathematics 2019(7), 153 (2019)

18. Gholizadeh, L., Karapınar, E.: Best proximity point results in dislocated metric spaces via R-functions. Rev. R. Acad. Cienc. Exactas Fís. Nat., Ser. A Mat. 112, 1391-1407 (2018)

19. Karapınar, E.: A note on Meir-Keeler contractions on dislocated quasi-b-metric. Filomat 31, 4305-4318 (2017)

20. Karapınar, E., Salimi, P.: Dislocated metric space to metric spaces with some fixed point theorems. Fixed Point Theory Appl. 2013(2013), 222 (2013)

21. Karapınar, E.: A short survey on dislocated metric spaces via fixed point theory. In: Banas, J., et al. (eds.) Advances in Nonlinear Analysis via the Concept of Measure of Noncompactness, pp. 457-483. Springer, Berlin (2017)

22. Chen, C., Dong, J., Zhu, C.: Some fixed point theorems in b-metric-like spaces. Fixed Point Theory Appl. 2015(2015), 122 (2015)

23. Rahman, M.U., Sarwar, M.: Dislocated quasi b-metric space and fixed point theorems. Electron. J. Math. Anal. Appl. 4 16-24 (2016)

24. Shahzad, N., Roldán López de Hierro, A.F., Khojasteh, F.: Some new fixed point theorems under $(\mathcal{A}, \mathcal{S})$-contractivity conditions. Rev. R. Acad. Cienc. Exactas Fís. Nat., Ser. A Mat. 111, 307-324 (2017)

25. Adigüzel, R.S., Aksoy, Ü., Karapınar, E., Erhan, I.M.: On the solution of a boundary value problem associated with a fractional differential equation. Math. Methods Appl. Sci. (2020). https://doi.org/10.1002/mma.6652

26. Al-Mezel, S.A., Alsulami, H.H., Karapınar, E., Roldán López de Hierro, A.F.: Discussion on "multidimensional coincidence points" via recent publications. Abstr. Appl. Anal. 2014, Article ID 287492 (2015)

27. Roldán López de Hierro, A.F., Karapınar, E., Manro, S.: Some new fixed point theorems in fuzzy metric spaces. J. Intell. Fuzzy Syst. 27, 2257-2264 (2014)

28. Proinov, P.D.: Fixed point theorems for generalized contractive mappings in metric spaces. J. Fixed Point Theory Appl. 22, Article ID 21 (2020)

29. Wardowski, D.: Fixed points of a new type of contractive mappings in complete metric spaces. Fixed Point Theory Appl. 2012, 94 (2012)

30. Jleli, M., Samet, B.: A new generalization of the Banach contraction principle. J. Inequal. Appl. 38, 1-8 (2014)

31. Karapınar, E., Roldán, A., Sadarangani, K.: Existence and uniqueness of best proximity points under rational contractivity conditions. Math. Slovaca 66, 1427-1442 (2016)

32. Dass, B.K., Gupta, S.: An extension of Banach contraction principle through rational expressions. Indian J. Pure Appl. Math. 6, 1455-1458 (1975)

33. Jaggi, D.S.: Some unique fixed point theorems. Indian J. Pure Appl. Math. 8, 223-230 (1977)

34. Hitzler, P., Seda, A.K.: Dislocated topologies. J. Electr. Eng. 51, 3-7 (2000)

35. Czerwik, S.: Contraction mappings in b-metric spaces. Acta Math. Inform. Univ. Ostrav. 1, 5-11 (1993)

36. Hussain, N., Roshan, J.R., Parvaneh, V., Abbas, M.: Common fixed point results for weak contractive mappings in ordered b-dislocated metric spaces with applications. J. Inequal. Appl. 2013(2013), 486 (2013) 